\title{
Familia y trabajo en México y Brasil
}

\author{
Brígida García, Humberto Muñoz y \\ Orlandina de Oliveira
}

\section{INTRODUCCIÓN}

En ESTE ARTículo presentamos un conjunto de consideraciones de orden teórico-metodológico sobre la participación económica familiar en áreas urbanas. Se trata de reflexiones hechas a partir de dos investigaciones: una en la ciudad de México y otra en Recife y São José dos Campos en Brasil, que ya están concluidas y sus resultados publicados. ${ }^{2}$ Regresamos a ellas en un intento por sistematizar lo hecho y tener una mejor apreciación de los

1 Una versión preliminar de este trabajo fue presentada en la conferencia, "Demographic Research in Latin America: Linking Individual, Household and Societal Variables", Social Science Research Council y Population Council, Ixtapan de la Sal, México, agosto, 1982.

2 Los resultados de las investigaciones se encuentran en: Brígida García, Humberto Muñoz y Orlandina de Oliveira, Hogares y Trabajadores en la Ciuded de México, El Colegio de México e Instituto de Investigaciones Sociales, Universidad Nacional Autónoma de México, (UNAM), 1982; y en Famiiia y Mercado de Trabajo (un estudio de dos ciudades brasileñas), El Colegio de México e Instituto de Investigaciones Sociales, UNAM, 1983 (en prensa). El estudio de México se basó en la información recolectada en la Encuesta de Migración Interna, Estructura Ocupacional y Movilidad Social en la Ciudad de México realizada por El Colegio de México y el Instituto de Investigaciones Sociales de la UN AM; el correspondiente a las ciudades brasileñas utilizó información de la Pesquisa Nacional de Reprodução Humana (PNRH), llevada a cabo por el Centro Brasileiro de Análise e Planejamento (CEBR AP). 
obstáculos que enfrentamos y de los avances logrados. Vemos en esta autocrítica una manera de estimular la discusión sobre una perspectiva de análisis que privilegia a la familia como categoría teórica y empírica en el estudio de diferentes aspectos de la fuerza de trabajo. ${ }^{3}$

Los planteamientos que hacemos se sitúan en diferentes niveles de abstracción y tocan problemas de índole diversa. Se articulan alrededor del intento que hicimos de vincular diferentes niveles de análisis (macroestructural, familiar e individual) y de relacionar varias dimensiones (la demográfica, social y económica) en el estudio de la actividad económica de los individuos.

Inicialmente explicamos el por qué de nuestro interés en el estudio de la participación económica familiar y la manera en que enfocamos su análisis. Señalamos también cómo el esquema analítico que utilizamos en la primera investigación realizada en la ciudad de México fue enriquecido con el análisis de las dos ciudades brasileñas. De ahí pasamos a los supuestos y a la conceptualización de unidad doméstica que orientaron la selección de los ejes ordenadores del estudio en el nivel familiar; presentamos los indicadores que utilizamos para acercarnos al concepto de contex to familiar y subrayamos algunas de sus limitaciones. También en esta sección dejamos claro el tipo de relación que planteamos entre la dimensión económica y la sociodemográfica de las 'unidades domésticas. Por último examinamos la manera en que introdujimos en el análisis la dinámica de la población y del mercado de trabajo como determinantes más generales de la participación económica familiar. A modo de consideración final mencionamos algunas de las limitaciones más generales del estilo de investigación analizado en este artículo.

Un último aspecto que queremos destacar es que los resultados concretos de las investigaciones aquí reseñadas son utilizados en este trabajo para diferentes propósitos. En algunos casos nos ayudan a destacar la utilidad analítica de algunos conceptos; en otros, nos permiten ilustrar las limitaciones de algunos indicadores transversales de conceptos dinámicos. También utilizamos los hallazgos para sugerir interpretaciones sobre la influencia de

3 Entre las investigaciones recientes, o en proceso, que se ubican en esta perspectiva están: De Barbieri (1982); Giner de los Rios (1982); Quesnel y Lerner: (1982); Margulis (1982); Martínez (1982); Martínez y Rendón (1982); Torres (1982). 
la dinámica poblacional en la estructura de las unidades domésticas y para demostrar el distinto papel que juega la familia en el condicionamiento de la participación económica de los individuos en diferentes contextos estructurales.

\section{LA PARTICIPACIÓN ECONÓMICA FAMILIAR}

En muchas ciudades latinoamericanas una familia promedio no consigue a menudo subsistir con el ingreso monetario de uno de sus miembros. En un marco estructural de deterioro del salario real y de deficiencia de los servicios colectivos se hace necesario conocer y especificar las alternativas a que recurren los trabajadores y sus familias para satisfacer sus necesidades básicas.

La literatura sobre el tema sugiere que estas alternativas pueden ser múltiples y que varían según la especificidad histórica de cada región o ciudad analizada. Por un lado, los miembros de las unidades domésticas pueden dedicar una mayor parte de su tiempo a algunas actividades realizadas en el ámbito del hogar que generan valores de uso fundamentales para su manutención (Oliveira, 1972; Jelin, 1974; De Barbieri, 1982; Singer, 1977). Estas actividades intradomésticas absorben parte del costo de reproducción de la fuerza de trabajo que no es cubierta por los ingresos monetarios de la unidad doméstica.

En la esfera extradoméstica los trabajadores organizados pueden, en coyunturas particulares, presionar por incrementos salariales y de prestaciones, vía seguro social u otros servicios que proporciona el Estado o el empleador en renglones como educación, salud, vivienda, transporte, etc. No obstante, esta alternativa sólo existe para quienes trabajan por contrato y están protegidos por la ley laboral; de ahí que muchas familias recurran a vínculos extradomésticos en épocas difíciles (desempleo, enfermedad, etc.). Estos vínculos incluyen ayudas de parientes, amigos o compadres y pueden asumir la forma de préstamos monetarios, comida, alojamiento o cuidado de los hijos (Lomnitz, 1975). Vinculados estrechamente a las alternativas anteriores, están los esfuerzos por incrementar los ingresos monetarios de los miembros del hogar que trabajan, siempre que existan las oportunidades para hacerlo. Se puede intentar ampliar la jornada de trabajo, o incluso obtener un segundo empleo. Sin 
embargo, estas estrategias pueden resultar infructuosas o insuficientes, y habrá necesidad de que los miembros de la familia que no trabajan en un momento determinado busquen un empleo (Schmink, 1979).

Las dos investigaciones que ahora comentamos se centran precisamente en esta última alternativa; en ellas analizamos el grado y la manera de incorporación de la mano de obra familiar (integrantes de los hogares que no son los jefes) en el mercado y sus múltiples condicionamientos. En el caso del estudio de la ciudad de México, privilegiamos las características económicas y sociodemográficas de las familias y la manera en que éstas influyen sobre los niveles de participación económica de sus miembros. En el análisis de las dos ciudades brasileñas, el énfãsis inicial en los procesos que ocurren en el ámbito familiar fue enriquecido por un mayor interés en ver cómo situaciones de mercado de trabajo muy distintas condicionan el grado en que las familias utilizan su mano de obra disponible.

La hipótesis general que subyace en el estudio de Brasil es que las condiciones de vida que enfrentan las familias y las posibilidades que se les presentan de elevar su ingreso a través de un incremento en la participación económica familiar depende de los rasgos sociodemográficos de las unidades domésticas y de sus integrantes, y del contexto estructural en que se encuentran inmersas. El tipo de empleo disponible y los niveles de remuneración asignados a cada uno de ellos inciden sobre las alternativas abiertas a los trabajadores para mantener o incrementar su nivel de vida, y por ende pueden modificar el impacto de los condicionamientos familiares $\mathrm{e}$ individuales ${ }^{4}$ sobre la participación económica de los distintos miembros de los hogares.

Por otra parte, un ángulo metodológico importante presente en los dos estudios de participación económica familiar fue la correspondencia que establecimos entre la unidad de referencia teórica y la unidad de análisis empírico. Basados en contribuciones sobre indicadores de dependencia económica en niveles agregados y familiares (Tienda, 1976), diseñamos una tasa de actividad por unidad doméstica; ésta relaciona el número de per-

4 Entre las características individuales incorporadas en nuestras investigaciones están: sexo, edad escolaridad y ubicación en la estructura de parentesco. Por estar ampliamente estudiada su importancia en las investigaciones sobre el tema, no las tratanos específicamente en este artículo. 
sonas, hombres o mujeres, de un determinado hogar que trabaja en el mercado con el número de personas del sexo correspondiente que existe en la misma unidad en edades activas.

Las tasas de participación familiar masculinas y femeninas, adultas y juveniles, nos permitieron analizar el grado de utilización de la mano de obra disponible en determinados grupos de unidades domésticas, identificados según algunas características sociodemográficas del jefe $\mathrm{y}$ aspectos de su estructura interna, tal como veremos a continuación.

\section{EJES ORDENADORES DEL ANÁLISIS EN EL NIVEL FAMILIAR}

La utilización del hogar ${ }^{5}$ como unidad de análisis de la participación de los individuos en la actividad económica trae implícita una determinada conceptualización. El hogar es visto como un ámbito social donde los individuos organizan, en armonía o en conflicto, diversas actividades necesarias para la reproduccion de la vida inmediata. Unos trabajan en el mercado para que otros estudien y otros se hacen cargo de las labores domésticas para que aquéllos trabajen fuera o estudien. Quienes no reciben ingresos necesitan de los recursos monetarios de los otros para subsistir, pero también los que lo reciben requieren de los bienes y servicios producidos en el ámbito doméstico, fundamentales para la manutención cotidiana (De Barbieri, 1982).

En suma, la pertenencia a un hogar supone una experiencia de vida común; de esta manera, cada miembro encuentra múltiples estímulos u obstáculos a su acción individual. Formar parte de una familia también implica utilizar una misma infraestructu-

5 Conviene aclarar que en la captación dê lá intúrmación para la ciudad de México el hogar-unidad doméstica fue definido como una unidad de consumo, esto es, el conjunto de individuos que habitan bajo un mismo techo, integran $y^{\prime}$ disfrutan de un presupuesto común. El término familia, en cambio, se utilizó de manera restringida para aquellos integrantes del hogar-unidad doméstica emparentados entre sí por vínculos de sangre, adopción o matrimonio. En la investigación de Brasil -Pesquisa Nacional de Reprodução Humana (PNRH) - se utilizó el término familia principal para lo que en México se definió como hogar-unidad doméstica, aproximadamente. Para facilitar la exposición, utilizaremos de aquí en adelante los diferentes términos como sinónimos para referirnos a la unidad de consumo que forman un conjunto de individuos, estando o no unidos por lazos de parentesco. 
ra, aunque de modo desigual, para la satisfacción de las necesidades materiales de existencia.

A partir de esta conceptualización general definimos en el análisis empírico dos ejes básicos -uno económico y otro sociodemográfico -- como condicionantes de la participación económica en el nivel familiar: la inserción laboral del jefe y las características sociodemográficas de las unidades. Estas dos dimensiones están relacionadas, pero también guardan su propia autonomía relativa; asimismo, son indicadores de las necesidades materiales básicas y de la disponibilidad de mano de obra en los hogares.

\section{El eje económico: la inserción laboral y la condición de hombre o mujer del jefe del hogar6}

Tomamos al jefe del hogar como punto de partida para estructurar el análisis de la participación familiar. La hipótesis central era que los individuos pertenecientes a un hogar comparten ìos beneficios o desventajas derivados de las condiciones económicas del jefe, y que la satisfacción de sus necesidades básicas dependen en buena medida de la posición de éste en la estructura económica y de su condición de hombre o mujer. Ambos aspectos se aunan para configurar una situación de escasez o de privilegio relativo para el jefe y su familia, y por ende inciden sobre el grado de utilización de la mano de obra disponible en el hogar.

En las ciudades latinoamericanas las familias se encuentran encabezadas habitualmente por hombres. A pesar del peso minoritario de las unidades dirigidas por mujeres, éstas son de gran interés sociodemográfico (Merrick y Schmink, 1978; Jelin 1978) y presentan rasgos de estructura interna bastante diferentes a las familias dirigidas por hombres: generalmente son más peque-

6 En la encuesta de la ciudad de México el jefe es la persona reconocida como tal por los demás niembros del hogar. En la encuesta de Brasil (PNRH) es posible distinguir entre el jefe del hogar reconocido por sus miembros como tal y el jefe conómico, o sea, aquel que entrega la mayor contribución económica al grupo doméstico. Utilizamos al jefe reconocido como eje del análisis porque en primer lugar, en casi todos los casos (nueve de cada diez), éste era a la vez el jefe económico; y además consideramos que desde un punto de vista sociológico la estabilidad y la solidez de los nexos familiares es mayor con el jefe reconocido que con el jefe económico. 
ñas y su composición de parentesco es mayoritariamente no nuclear (Burch, Lira y Lopes, 1976). Tanto en el estudio de México como en el Brasil, el sexo del jefe constituyó una característica clave en el análisis de los rasgos sociodemográficos de las unidades.

Sin embargo, fue en el estudio de las dos ciudades brasileñas donde pudimos establecer con mayor claridad la necesidad de incorporar la condición de hombre o mujer del jefe en el análisis de la participación económica familiar; su inclusión resultó de gran utilidad analítica: encontramos, sin excepciones, que hombres y mujeres adultas y los jóvenes de uno y otro sexo trabajan con mayor frecuencia cuando viven en familias dirigidas por mujeres.

Respecto a la inserción laboral de los jefes recurrimos al uso de clasificaciones que captan divisiones sustanciales entre los trabajadores. El primer criterio empleado fue si vendían o no su fuerza de trabajo; aquí distinguimos a los asalariados de los trabajadores por cuenta propia: los primeros son los mayoritarios en las tres ciudades analizadas, pero los que trabajan por cuenta propia todavía representan una fracción importante de su fuerza de trabajo como en otras ciudades latinoamericanas, pues no siempre tienden a desaparecer a medida que avanza la implantación de formas capitalistas de producción (Jelin, 1967; Muñoz y Oliveira, 1976; Prandi, 1978). De ahí que sea importante, en estudios como los nuestros, conocer si las fanilias de jefes trabajadores por cuenta propia abastecen o no de fuerza de trabajo asalariado al mercado. En el caso de la ciudad de México, verificamos que la mayor parte de la mano de obra familiar que sale de hogares de jefes por cuenta propia se encuentra trabajando como asalariada.

En el caso de los trabajadores asalariados, tuvimos en cuenta que la división social y técnica del trabajo impone entre ellos distinciones objetivas. El capital subordina al trabajo en formas muy variadas (Braverman, 1974); lo fracciona a su conveniencia buscando mantener bajos los salarios, crea jerarquías a través de las condiciones de trabajo y de esa manera surgen contenidos ideológicos que hacen creer a unos trabajadores que son superiores a los otros. Por lo anterior, diferenciamos a los asalariados en trabajadores manuales y no manuales. La situación privilegiada de estos últimos tiene como expresión más evidente el prestigio 
social de las ocupaciones que ejercen y el mayor nivel de escolaridad que por lo regular exige el desempeño de tales ocupaciones. De ahi la tendencia a que el trabajo no manual sea mejor remunerado.

Hay, desde luego, muchas otras maneras y propósitos de captar la división entre los asalariados (Przeworski, 1978). En lo que se refiere a nuestros objetivos analiticos concretos, la diferenciación manual-no manual se constituyó en un aspecto clave del estudio de la participación económica familiar.

Es importante aclarar que la inserción laboral de los jefes de familia fue establecida a partir de información en un momento en el tiempo; es decir, no tomamos en cuenta su trayectoria laboral por no contar con la información necesaria, lo cual no significa que esta trayectoria no esté relacionada con las características de algunos miembros del hogar y por ende con su participación en la actividad económica. Así, por ejemplo, un jefe que entró y salio de la actividad económica múltiples veces a lo largo de su vida laboral (por desempleo, enfermedad, etc.), y que sólo al final de la misma consigue un empleo estable con una remuneración por encima del salario mínimo, posiblemente no ha tenido las condiciones económicas mínimas para educar a sus hijos, a no ser que otros miembros del hogar hayan entrado en escena para garantizar con su trabajo esta capacitación. Un jefe con la misma inserción laboral, pero con una trayectoria ocupacional más estable, seguramente habrá podido, aunque con sacrificios, educar a sus hijos. Los distintos niveles educativos de los hijos en uno u otro caso pueden configurar para ellos diferentes alternativas de participación en el mercado de trabajo.

\section{El eje sociodemográfico: la estructura interna de las familias}

El estudio de las unidades domésticas en términos de su estructura interna fue un paso necesario para el análisis de la participación económica familiar; seleccionamos la composición de parentesco, el ciclo vital y el tamaño de los hogares para tal propósito. Estos son algunos de los aspectos que se consideran clave dentro de la demografía de la familia (Burch, Lira y Lopes, 1976) y son para nosotros de gran interés porque inciden en la estructura por edad y sexo del hogar y, en consecuencia, en el 
monto y características de los integrantes disponibles para trabajar y en el número de dependientes.

En lo que se refiere a la composición de parentesco, utilizamos la diferenciación entre unidades domésticas nucleares, extendidas y compuestas, que constituyen clasificaciones comúnmente utilizadas en muchas investigaciones. Además, incorporamos la modalidad "sin componente nuclear" que agrupa a las unidades en las que el jefe no tiene pareja ni hijos solteros.

La composición de parentesco, y también el ciclo vital y el tamaño, son rasgos esencialmente dinámicos. Se sostiene a menudo que las unidades extendidas son apenas una fase por la que atraviesan las nucleares. ${ }^{7} \mathrm{El}$ ciclo vital, por su parte, hace esencialmente alusión a los estadios por los que las familias atraviesan a partir de su formación (Kono, 1977). El tamaño también sufre transformaciones importantes en el tiempo, dependiendo, por supuesto, de la composición de parentesco y del ciclo vital en cuestión.

Por este carácter dinámico las características sociodemográficas de las unidades presentan problemas de captación adecuada en un análisis de tipo transversal. El caso más claro en este sentido es al del ciclo vital.

En el estudio de la ciudad de México escogimos como indicador de ciclo vital la edad del jefe del hogar, y especificamos, en vez de dar por supuestas, las relaciones de este indicador con la composición de parentesco, el tamaño y la composición por cdad de los hogares. En las ciudades brasileñas refinamos el indicador de ciclo vital y combinamos la edad de los jefes con las edades de los hijos mayores debido a nuestro interés en conocer la disponibilidad de mano de obra presente en las unidades domésticas. Esta alternativa resultó exitosa desde numerosos puntos de vista, pero también nos condujo a otra clase de problemas, pues solo conocíamos los hijos residentes en el hogar.

El no contar con información sobre los hijos ausentes puede llevar a clasificaciones erróneas del ciclo vital, sobre todo si se comparan sectores donde la partida de los hijos del hogar paterno puede ser más temprana en uno que en otros. Esto lo vimos al

7 Existe alguna evidencia indirecta de que esto sucede en alguna medida en la ciudad de México, ya que, al analizar la composición de parentesco de las unidades por grupos de edad de los jefes, las extendidas aumentaron su peso a medida que las edades eran mayores. 
comparar los hogares de los jefes manuales con los de los jefes no manuales. La mayor pobreza relativa de los jefes manuales puede llevar a que sus hijos salgan más temprano de la casa (sea porque emigren o porque forman otra familia). Este proceso llevaría a un aparente "rejuvenecimiento" de las familias nucleares de los jefes manuales frente a las de los no manuales, que retienen a sus hijos en la casa por más tiempo. En suma, el indicador más refinado puso aún más en evidencia los problemas derivados de la información transversal de los procesos dinámicos. Sin embargo, nos proporcionó muchos elementos para enriquecer nuestra visión de los rasgos de las unidades domésticas como cristalizaciones de una serie de procesos sociales y demográficos.

\section{Interrelación entre lo económico y lo demográfico en el nivel familiar}

En los dos estudios caracterizamos las unidades domésticas en términos de su dimensión económica y sociodemográfica. Pero queremos destacar que no nos interesaba aislar el peso relativo que tiene la inserción laboral de los jefes en comparación con las características sociodemográficas de sus hogares en el condicionamiento de la participación económica familiar. Partimos de la idea de que es más importante estudiar la manera en que ambos aspectos interactúan para explicar el fenómeno analizado y por ello combinamos los dos ejes y llegamos al concepto de contex to familiar.

Desde esta perspectiva, para el análisis de la participación familiar combinamos el ciclo vital y la composición de parentesco con la inserción laboral del jefe. La agrupación de los agregados de hogares en contextos claramente diferenciados, nos permitió tomar en cuenta los múltiples condicionamientos de la participación económica que operan en el ámbito familiar.

Para configurar los contex tos familiares analizamos las variaciones en las estructuras internas de las familias encabezadas por trabajadores asalariados manuales y no manuales y por trabajadores por cuenta propia, pero no establecimos una relación de causalidad entre la inserción laboral del jefe y las características sociodemográficas de sus familias. Como ilustraremos en la próxima sección, estas últimas sintetizan la dinámica demográfica 
existente en las áreas urbanas analizadas, y no tienen por qué ser conceptualizadas exclusivamente como resultantes de las condjciones económicas que prevalecen en un momento determinado.

Conviene también explicitar que optamos por definir contextos familiares porque desde un punto de vista conceptual, ambos ejes - la inserción laboral del jefe y las características sociodemográficas de los hogares- no actúan de manera aislada y unidireccional sobre los niveles de participación económica familiar, ambos influyen en la configuración de la oferta de mano de obra así como en la definición de las necesidades básicas, aspectos que a su vez inciden sobre los niveles de participación. Por ejemplo, aun cuando aceptemos que no existe una misma pauta de necesidades para todos los sectores sociales, es razonable plantear que su magnitud y naturaleza varía de acuerdo con las propias características sociodemográficas de los hogares, principalmente con su tamaño y con la carga de dependientes. La situación económica del jefe puede afectar la disponibilidad de mano de obra a través de la contratación de empleadas domésticas, por ejemplo. Con ello se libera a las esposas, y en ocasiones a las hijas, de los quehaceres del hogar. Quedan así disponibles mujeres para incorporarse a la actividad económica o para dedicarse a los estudios.

En este contexto, es ilustrativo el caso de las familias dirigidas por asalariados no manuales. Allí vimos cómo los rasgos económicos y los sociodemográficos se mezclan y condicionan niveles diferenciales de participación para los diversos integrantes del hogar. Veamos un ejemplo. En las tres ciudades estudiadas, estas unidades comparten una serie de rasgos que, en alguna medida, explican la escasa participación de sus hombres y mujeres jóvenes, por un lado, y la elevada participación de las mujeres y hombres adultos por el otro. Estas son las unidades de menor tamaño relativo y dirigidas por jefes con los promedios más altos de ingreso. Además, casi siempre son hogares que cuentan con servicio doméstico remunerado. Todos estos aspectos propician que las mujeres adultas se incorporen al mercado de trabajo, sobre todo si tienen elevada escolaridad y el ciclo vital familiar está avanzado.

Las características económicas y sociodemográficas de estas familias facilitan también que se desarrolle una estrategia de calificación de los miembros más jóvenes, en especial de los 
varones, con miras a asegurar en el futuro su posición en la sociedad. Hay alguna información que documenta lo anterior para México (Valle, 1980) y para Brasil (Doria-Bilac, 1978). Es una estrategia que, en algunos casos, puede involucrar la participación de uno o varios miembros adultos además del jefe del hogar para el beneficio de las nuevas generaciones.

\section{Condicionantes macroestructurales}

\section{Dinámica poblacional y familia}

Reconstruimos la dinámica demográfica y socioeconómica de las ciudades que estudiamos para contar con los elementos que nos permitieran entender, entre otros aspectos, las características de las familias y de la participación económica de sus miembros.

Así, vimos que las áreas urbanas estudiadas mantienen todavía una alta fecundidad, relativamente baja mortalidad y son recipientes de un intenso proceso migratorio. Esto lleva a configurar un tamaño promedio de familia elevado y un amplio conjunto de hogares en las etapas tempranas del ciclo vital con hijos pequeños. Como encontramos además que la mayor parte de las unidades es nuclear, estamos frente a familias que por lo común cuentan con escasa disponibilidad de mano de obra y baja participación económica familiar, a pesar de lo apremiante que puedan ser las necesidades económicas. La esposa es casi siempre la otra persona en edad activa en el hogar nuclear del ciclo joven, pero, en muchas ocasiones tiene que dedicarse a la realización del trabajo doméstico.

Esta situación de escasez de mano de obra cambia cuando el ciclo vital es avanzado y en las unidades no nucleares. Existe en las tres ciudades una proporción no despreciable de arreglos extendidos, compuestos y "sin componente nuclear". En todas estas unidades, a diferencia de las nucleares jóvenes, los hijos adultos $\mathrm{u}$ ołros parientes del jefe pueden trabajar o hacerse cargo de las tareas del hogar para que otros trabajen y asi hacer frente a la presión económica. Nosotros encontramos que la participación económica de los miembros de las familias adquiere niveles más elevados en las unidades nucleares de ciclo avanzado y en 
las extendidas y compuestas, en especial entre los sectores menos privilegiados de la sociedad.

Entre los factores que influyen en la formación de unidades no nucleares están la mortalidad diferencial por sexo, la migración y las pautas de nupcialidad imperantes. La viudez es más acentuada entre la población femenina y ocurre frecuentemente que al morir el esposo, la mujer se traslada y uno de sus hijos casados, o un hijo soltero pasa a ser el jefe del hogar. En ambos casos la familia nuclear se transforma en no nuclear. Existen varios estudios en Brasil que documentan este mecanismo (DoriaBilac, 1978).

La migración contribuye a la formación de hogares no nucleares en áreas urbanas de atracción poblacional porque una buena parte de los migrantes llegan solos y se van a vivir a casa de parientes o amigos. Cuando la familia receptora del nuevo migrante es nuclear se transforma en extendida o compuesta. Hay registros de que este proceso ocurre por lo menos en dos de las áreas urbanas que analizamos (Muñoz, Oliveira, Stern, 1977; Martins Rodrigues, 1979).

Por otra parte, hay que agregar que la composición de parentesco de las familias también depende de procesos económicos. Aquí solamente ilustraremos el punto para no extendernos. Por ejemplo, la escasez de vivienda y las dificultades que enfrentan los jóvenes para encontrar empleo o percibir salarios remuneradores influyen para que las nuevas parejas permanezcan en el hogar de los padres. De esa manera, los factores económicos inciden sobre la formación de unidades extendidas "típicas" de gran tamaño: a veces encontramos hasta tres generaciones bajo un mismo techo.

Otro ejemplo de interés para ver cómo operan los procesos demográficos sobre la estructura de la familia es el caso de las unidades encabezadas por mujeres, que también son contextos que se caracterizan por elevados niveles de participación económica familiar. La existencia de hogares con jefas es resultado de un conjunto de factores. Uno de ellos es la mayor esperanza de vida de las mujeres, a lo que se agrega que muy pocas viudas se vuelvan a casar. Además, en determinadas regiones, como en el noreste brasileño, el celibato femenino y las tasas de separación son mayores que en otros puntos del país (Goldani y Wong, 1981), lo que se vincula con el déficit de hombres que muestran 
los datos del censo y con la mayor incidencia de hogares dirigidos por mujeres en ciudades como Recife.

\section{Participación económica familiar en diferentes mercados de trabajo}

En el estudio de la ciudad de Méxíco incorporamos la dinámica del mercado de trabajo como telón de fondo para interpretar los resultados encontrados en el nivel familiar. Esto nos llevó a identificar y a analizar varios factores que podrian estar condicionando la participación económica familiar. Entre ellos destacamos: a) crecimiento del empleo en las dos décadas anteriores al estudio (1950-1970), aunque los ritmos no fueron tan acelerados como en el período $1930-1950 ; b$ ) importante creación de ocupaciones no manuales (técnicas y profesionales); $c$ ) una creciente proletarización de la mano de obra y un incremento en las exigencias de contratación particularmente en lo que se refiere a la escolaridad; y $d$ ) la persistencia de fuertes desigualdades socioeconómicas entre los diversos sectores de trabajadores.

En un marco como el descrito es explicable que hayamos encontrado modalidades distintas de participación familiar al comparar unidades domésticas pertenecientes a diferentes sectores sociales. Para las familias dirigidas por trabajadores asalariados manuales reportamos tasas masculinas adultas y juveniles que están entre las más elevadas de todos los casos estudiados. Aquí se trata de hombres jóvenes y adultos que viven en familias de gran tamaño cuyos jefes reciben muy baja remuneración; es decir hogares con disponibilidad de mano de obra pero con muchos miembros para mantener con el parco salario de un solo trabajador. También encontramos situaciones familiares como la de los jefes no manuales, que se caracterizan por una alta participación femenina; se trata de mujeres con una elevada escolaridad. En este caso también aparece con claridad cómo la modernización del terciario, la ampliación de sectores no manuales y el mayor acceso a oportunidades educativas contribuyen a aumentar la participación económica femenina.

Fue en el estudio de Brasil donde pudimos analizar el impacto de los mercados de trabajo sobre la participación junto con la influencia derivada de la situación familiar. Alli contábamos con 
dos áreas urbanas (Recife y Sảo José dos Campos) que se distinguen por su proceso histórico de formación, tamaño, localización geográfica regional, función en la red urbana del país, tendencias sectoriales del empleo y dinámica demográfica. Mediante este recurso de análisis comparativo de dos situaciones estructurales distintas, con economías predominantemente capitalistas pudimos acercanos a la especificidad del fenómeno y a la importancia relativa que tienen los diversos factores explicativos

En general encontramos que las características estructurales de los mercados de trabajo son las que definen el monto de la participación familiar en la actividad: para la ciudad paulista de São José dos Campos reportamos, casi siempre, niveles de participación familiar sistemáticamente más elevados que los encontrados en Recife. En la ciudad paulista, la penetración del capital de corte oligopólico ha creado una gran cantidad de empleos gracias a la instalación y rápido crecimiento del parque industrial y de los servicios. Se trata de una economía urbana con necesidades de mano de obra que reflejan el movimiento expansivo del capital el cual requiere que varios miembros de las familias se incorporen a la actividad. Esta es, a su vez, una forma de presionar hacia abajo la estructura salarial; se utilizan trabajadores (mujeres o jóvenes) cuya remuneración puede establecerse más fácilmente por debajo del costo de reproducción de su fuerza de trabajo.

Son los integrantes de familias más pobres, de mayor tamaño relativo y no nucleares las que responden de manera más evidente a esta dinámica económica. Se trata de unidades domésticas dirigidas por trabajadores por cuenta propia y asalariados manuales. Sin embargo, aun los miembros de contextos familiares poco propicios a la participación económica (hogares nucleares jóvenes con escasa disponibilidad de fuerza de trabajo para la realización del trabajo doméstico) presentan niveles elevados de participación económica. Con base en estos resultados podemos afirmar que el papel de la situación familiar es más restringido en São José dos Campos que en Recife, como veremos a continuación. Asimismo, en el caso de la primera ciudad estamos frente a un contex to global de desarrollo urbano donde la incorporación de mujeres y adolescentes al mercado de trabajo cumple seguramente un doble papel: contribuye a la depreciación de los salarios al incrementar la oferta de mano de obra. pero también a elevar el 
nivel de vida individual o familiar (aunque sea con la suma de bajos salarios). Vistas las cosas de otra manera, tenemos un incremento de la renta familiar que tiene lugar a través de la explotación de mayor cantidad de mano de obra con bajos salarios.

Recife, por el contrario, es representativa del tipo de economía urbana en que la penetración del capitalismo industrial en años recientes ha tenido como consecuencia una modesta creación de empleos. Sin embargo, como capital estatal, Recife ha tenido una expansión importante del sector terciario. Coexisten en su estructura ocupacional trabajadores asalariados manuales y no manuales, además de un importante contingente de trabajadores por cuenta propia.

A pesar de la estrechez económica que caracteriza a muchos grupos de hogares en Recife, la mano de obra familiar sólo consigue contribuir a aliviar la penuria existente en un número determinado de situaciones. Es ilustrativo en este respecto el caso de las mujeres adultas que presentan niveles de ocupación relativamente elevados en esta ciudad. Por el lado de la situación familiar, estas mujeres se lanzan al mercado de trabajo principalmente cuando cuentan con ayuda para el trabajo doméstico o cuando sus hijos ya han crecido como sucede en las unidades de ciclo avanzado o no nucleares. Por el lado del mercado, las mujeres que provienen de hogares con carencias económicas cuentan con alternativas abiertas en los sectores de servicios individuales, particularmente en actividades manuales no calificadas como el servicio doméstico, y en actividades por cuenta propia, como la venta ambulante, el arreglo de ropas, la preparación de alimentos, etc. Esto porque, en Recife, al igual que en otras ciudades latinoamericanas (Muñoz y Oliveira, 1979), la ampliación y la modernización de algunas actividades terciarias ocurrió aunada a la permanencia de otros servicios que reproducen la pobreza ingente pero son de gran importancia como fuente de empleo y de ingreso para la mujer adulta.

La mano de obra joven que vive en hogares pobres tiene pocas alternativas en el mercado de trabajo de Recife, a no ser que sean hijos $u$ otros parientes del jefe por cuenta propia que puedan ayudarlo en el negocio familiar. Son hijas que venden, hijos o parientes que efectúan las instalaciones a la vez que se capacitan en el oficio, etc.

Estos ejemplos son teóricamente relevantes porque ilustran 
cómo el orden social y el familiar se interpenetran, y cómo los rasgos socioeconómicos y demográficos de la unidad doméstica cobran más relevancia en unas situaciones que en otras para explicar la participación de la mano de obra familiar en el mercado de trabajo. Es así como podemos hablar de la familia como mediación; es decir, una instancia que reelabora el impacto de los procesos estructurales sobre la acción individual.

\section{UNA CONSIDERACIÓN FINAL}

La práctica de investigación que hemos tenido, y que reseñamos a lo largo de estas páginas, constituye un acercamiento analítico particular a un problema concreto. Esta experiencia ofrece potencialidades y también muchas limitaciones. A manera de consideración final mencionaremos algunas de estas últimas.

La participación económica familiar es un fenómeno que presenta un doble interés analítico. Es relevante desde un punto de vista teórico-metodológico ya que el examen de sus múltiples condicionamientos nos ha permitido explorar la articulación de diferentes niveles de análisis y, constituye un aspecto central de la vida cotidiana de quienes enfrentan condiciones difíciles en las ciudades latinoamericanas de hoy.

$\mathrm{Al}$ analizar la participación económica familiar estábamos interesados en buscar explicaciones a las regularidades encontradas en distintas áreas urbanas y en captar diferencias específicas entre ciudades como una manera de enriquecer el conocimiento del fenómeno. Por ello, a lo largo de este artículo hemos destacado algunas de las similitudes y diferencias encontradas y hemos dado ejemplos de la red de condicionantes en la que la participación económica familiar está inmersa.

Algunos de estos condicionantes fueron incorporados como referencias conductoras del análisis (como fue el caso del impacto de los procesos demográficos y socioeconómicos sobre los rasgos de los grupos domésticos); otros fueron objeto de estudio propiamente tal (como la dinámica de los mercados de trabajo y las características de los hogares y de sus jefes).

Ahora bien, pudiera ser objetable el recorte de la realidad impuesto por el tipo de datos que manejamos: restringimos nuestro objeto de estudio a la participación económica. Esta- 
mos seguros de que la comprensión de la reproducción de la vida cotidiana se enriquecería con el análisis de las múltiples conexiones entre participación económica familiar y otras actividades que desarrollan los miembros de los hogares como el trabajo doméstico, la producción de subsistencia, las redes de intercambio y ayudas entre vecinos y parientes.

Además, hay factores estructurales que afectan la participación económica familiar y que nosotros no hemos podido tomar en cuenta. En particular, sería importante conocer cómo funcionan los servicios que reciben los trabajadores a modo de salarios indirectos, la oferta de viviendas, etc. Asimismo, pudiera ser relevante saber en qué medida el Estado interviene en el funcionamiento del mercado laboral, sea como agente económico, como entidad jurídica que promulga leyes que regulan la contratación del trabajo o como rector de la política económica y demográfica. En suma, estudiar la parte que le toca al Estado para garantizar las condiciones generales de la producción capitalista, incluida la disminución en el costo de reproducción de la fuerza de trabajo.

En otro orden de consideraciones podemos afirmar que la combinación de datos globales provenientes de censos con datos de encuestas permitió enriquecer el análisis, pero no nos auxilió en la discusión de numerosos supuestos con respecto a los hogares. Los casos más ilustrativos en este sentido son la consideración de la residencia como elemento clave en la configuración de las unidades domésticas y la estructuración del análisis en torno a la figura del jefe reconocido como tal.

Estos supuestos podrían ser transformados en hipótesis de trabajo. Podríamos preguntarnos, como ya lo han hecho otros investigadores, en qué medida la familia residencial actúa como un eje organizador de la vida cotidiana de los individuos, o qué significa ser jefe en diferentes tipos de familias. Interrogantes como éstas llevan a la delimitación de nuevos problemas por investigar y requieren de un acercamiento a la realidad muy distinto del nuestro, como sería una mayor vinculación entre el proceso de captación y construcción de la información. Este tipo de acercamiento puede llevarse a cabo en estudios de caso, utilizando observación y entrevistas abiertas que son de gran interés; permite el ir y venir de los conceptos a los datos y enriquecer los primeros a lo largo del propio proceso de investiga- 
ción. Nosotros, al partir de información ya recolectada con base en definiciones establecidas a priori, ${ }^{8}$ tuvimos que precisar y limitar nuestro objeto de estudio y contar con márgenes mucho más estrechos para la reconstrucción, según nuestros intereses teóricos, de la realidad analizada.

\section{Bibliografía}

Braverman, Harry, Labor and Monopoly Capital. The Degradation of Work in the Twentieth Century, New York and London, Monthly Review Press, 1974.

Burch, Thomas, Luis F. Lira y Valdecir Lopes (editores), La familia como unidad de estudio demográfico, San José, Costa Rica, Centro Latinoamericano de Demografía (CELADE), 1976.

De Barbieri, Ma. Teresita, Mujeres y vida cotidiana (Estudio exploratorio en sectores medio y obreros de la ciudad de México), México, Instituto de Investigaciones Sociales de la Universidad Autónoma de México, (en prensa), 1982.

Doria-Bilac, Elizabeth, Familias de trabalhadores: estrategias de sobreviviencia, Coleção Ensaio e Memoria 6 São Paulo, Brasil, Ediçōes Símbolo, 1978.

García, Brígida, Humberto Muñoz y Orlandina de Oliveira, Hogares y trabajadores en la ciudad de México, México, El Colegio. đe México e Instituto de Investigaciones Sociales, Universidad Nacional Autónoma de México, 1982.

García, Brígida, Humberto Muñoz y Orlandina de Oliveira, Familia y mercado de trabajo, (un estudio de dos ciudades brasileñas), El Colegio de México (en prensa), 1983.

Giner de los Ríos, Francisco, "Microindustria y unidad doméstica, trabajo presentado al seminario sobre Grupos domésticos, familia y sociedad, [Centro de Estudios Sociológicos], El Colegio de México, 1982.

Goldani, Altmann, Ana María y Laura Rodríguez Wong, "Padroes e tendencias da nupcialidade no Brasil", Anais Segundo Encontro Nacional, Aguas de São Pedro, Brasil, Associação Brasileira de Estudos Populacionaes (ABEP), pp. 343-415, 1981.

Jelin, Elizabeth, "Secuencias ocupacionales y cambio estructural: historias de trabajadores por cuenta propia", en Las historias de vida en ciencias sociales. Teoria y técnica, Buenos Aires, Argentina, Ediciones Nueva Visión (Cuadernos de Investigación Social), 1967.

Jelin, Elizabeth, "La bahiana en la fuerza de trabajo: actividad doméstica, producción simple y trabajo asalariado en Salvador, Brasil", en Demografia y Economia, vol. VII, núm. 3, México, Centro de Estudios Económicos y Demográficos, El Colegio de México, pp. 307-321, 1974.

8 En ambos estudios se utilizaron datos de encuestas recolectados con otros objetivos centrales. Véase la nota 2 . 
Jelin, Elizabeth, "La mujer y el mercado de trabajo urbano", en Estudios CEDES, vol. I, núm. 6, Buenos Aires, Argentina, Centro de Estudios de Estado y Sociedad (CEDES), 1978.

Kono, Shigemi, "The Concept of the Family Life Cycle as a Bridge between Demography and Sociology, en International Population Conference, México, International Union for the Scientific Study of Population, pp. 355-370, 1977.

Lomnitz, Larissa, Cómo sobreviven los marginados, México, Siglo XXI Editores, 1975.

Margulis, Mario, "Reproducción de la unidad doméstica, fuerza de trabajo y relaciones de producción", trabajo presentado al seminario Grupos domésticos, familia y sociedad, [Centro de Estudios Sociológicos], E1 Colegio de México, 1982.

Martínez, Alicia Inés, "Reproducción cotidiana y reproducción social: una aproximación metodológica", trabajo presentado al seminario Grupos domésticos, familia y sociedad, [Centro de Estudios Sociológicos], El Colegio de México, 1982.

Martínez, Marielle y Teresa Rendón, "Reflexiones a partir de una investigación sobre grupos domésticos campesinos y sus estrategias de reproducción", trabajo presentado al seminario Grupos domésticos, familia y sociedad, [Centro de Estudios Sociológicos], El Colegio de México, 1982.

Martins Rodrigues, Jessita, $A$ mulher operaria: um estudo sobre tecelas, São Paulo, (Brasil), Editora Hucitec, 1979.

Merrick, Thomas, Marianne Schmink, "Female Headed Households and Urban Poverty in Brasil", documento presentado en el taller Women in Poverty: What do we know? Belmont Conference Center, abril, 1978.

Muñoz, Humberto y Orlandina de Oliveira, "Migración, oportunidades de empleo y diferencias de ingreso en la ciudad de México", en Revista Mexicana de Sociologia, año XXXVIII, vol. XXXVIII, núm. 1, México, 1976.

Muñoz, Humberto y Orlandina de Oliveira, "Algunas controversias sobre la fuerza de trabajo en América Latina" en [Rubén Katsman y José Luis Reyna (editores)] Fuerza de trabajo y movimientos laborales en América Latina, México, E1 Colegio de México.

Muñoz, Humberto, Orlandina de Oliveira y Claudio Stern, Migración y desigualdad social en la ciudad de México, México, Instituto de Investigaciones Sociales, Universidad Nacional Autónoma de México y El Colegio de México, 1977.

Oliveira, Francisco, "A economía brasileira: crítica a razão dualista", en Estudos CEBRAP, 2, 1972.

Prandi, José Reginaldo, O Trabalhador por conta propia sob o capital (Coleção ensaio e memoria 14), São Paulo, Brasil, Edições Símbolo, 1978.

Przeworski, Adam, "El proceso de la formación de clases", en Revista Mexicana de Sociología, año XL, núm. extraordinario (E), 1978.

Quesnel, André y Susana Lerner, "El espacio familiar en la reproducción social; grupos domésticos residenciales y grupos de interacción, algunas 
reflexiones a partir del estudio de la zona henequenera", trabajo presentado al seminario Grupos domésticos, familia y sociedad, [Centro de Estudios Sociológicos], El Colegio de México, 1982.

Schmink, Marianne, "Community in Ascendance: Urban Industrial Growth and Household Income Strategies in Belo Horizonte, Brazil", Tesis doctoral, Austin, Universidad de Texas, 1979.

Singer, Paulo, Economia politica do trabalho, São Paulo, Editora Hucitec, 1977.

Tienda, Marta, "Macro and Micro Contexts of Age and Economic Dependency: An Assessment with Peruvian Data", tesis de Doctorado, Austin, Universidad de Texas, 1976.

Torres, Mario, "Una aplicación empírica de un enfoque de reproducción social", trabajo presentado al seminario Grupos domésticos, familia y sociedad, [Centro de Estudios Sociológicos], El Colegio de México, 1982.

Valle Flores, María de los Angeles, "Oportunidades educativas y de participación económica de las mujeres en la ciudad de México", tesis de Licenciatura en Sociología, México, Facultad de Ciencias Políticas y Sociales, Universidad Autónoma de México, 1980. 
